\title{
Nonlocally related PDE systems for one-dimensional nonlinear elastodynamics
}

\author{
G. Bluman · A. F. Cheviakov · J.-F. Ganghoffer
}

Received: 5 July 2006 / Accepted: 15 February 2008 / Published online: 22 March 2008

(C) Springer Science+Business Media B.V. 2008

\begin{abstract}
Complete dynamical PDE systems of one-dimensional nonlinear elasticity satisfying the principle of material frame indifference are derived in Eulerian and Lagrangian formulations. These systems are considered within the framework of equivalent nonlocally related PDE systems. Consequently, a direct relation between the Euler and Lagrange systems is obtained. Moreover, other equivalent PDE systems nonlocally related to both of these familiar systems are obtained. Point symmetries of three of these nonlocally related PDE systems of nonlinear elasticity are classified with respect to constitutive and loading functions. Consequently, new symmetries are computed that are: nonlocal for the Euler system and local for the Lagrange system; local for the Euler system and nonlocal for the Lagrange system; nonlocal for both the Euler and Lagrange systems. For realistic constitutive functions and boundary conditions, new dynamical solutions are constructed for the Euler system that only arise as symmetry reductions from invariance under nonlocal symmetries.
\end{abstract}

Keywords Nonlinear elasticity $\cdot$ Nonlocal symmetries $\cdot$ Nonlocally related systems $\cdot$ Group invariant solutions

\section{Introduction}

Analytical studies of various linear and nonlinear, static and dynamic elasticity models, in particular, the problem of finding exact solutions, have attracted significant attention of researchers in recent years. Lie-group analysis is an important method for systematically studying systems of differential equations (DEs) and boundary-value problems. For a given DE problem, one can algorithmically calculate its admitted point symmetries - transformations of dependent and independent variables that map a problem into itself (e.g. [1-4]). Knowledge of admitted symmetries allows one to construct mappings relating DE systems, find out whether or not a given nonlinear DE system can be mapped into a linear system by an invertible transformation, and find exact (group-invariant or symmetry-generated)

G. Bluman $(\varangle)$ · A. F. Cheviakov

Department of Mathematics, University of British Columbia, Vancouver, Canada V6T 1Z2

e-mail: bluman@math.ubc.ca

A. F. Cheviakov

e-mail: alexch@math.ubc.ca

J.-F. Ganghoffer

LEMTA - ENSEM, 2, Avenue de la Foret de Haye, BP 160, 54504 Vandoeuvre les Nancy, Cedex, France

e-mail: jean-francois.ganghoffer@ensem.inpl-nancy.fr 
solutions. Lie-group analysis is of further interest in setting up numerical schemes preserving the group properties of a boundary-value problem (BVP) [5].

Lie symmetries are widely used in the analysis of contemporary elasticity models. In particular, Lie transformations have been applied to the classical linear theories of rods and plates [6]; the classification of Lie point symmetries for 1D and 2D nonlocal elastodynamics appears in [7, 8]. Isovector fields for the equations of homogeneous hyperelasticity written in Lagrangian form (excluding mass conservation) were presented in [9].

Similarity (invariant) solutions, arising from symmetry reduction, have been found for many linear elasticity problems. Similarity solutions of the Navier equations for linear elastodynamics problems have been obtained in closed-form in [10]. Similarity solutions for the motion of hyperelastic solids have been determined in [11, 12]. Focusing on nonlinear elasticity, it is well known that very few closed-form solutions of BVPs for compressible elasticity have been obtained in the literature (contrary to incompressible elasticity), due to the absence of the kinematic incompressibility constraint, as pointed out in $[13,14]$.

The problem of finding conservation laws (full divergence expressions) that hold for a system of partial differential equations (PDEs) is an important counterpart to symmetry analysis. In elasticity theory, one well-known application of conservation laws is the Eshelby energy-momentum tensor, and a related path-independent integral, which governs the energy release rate at a singularity [15]. In a series of papers, Olver studied conservation laws and related path-independent integrals in linear elastostatics within the framework of hyperelasticity (see [16] and references therein). In [17], conservation laws of elasticity equations are discussed from a geometric point of view. Other applications of conservation laws include the development and analysis of numerical methods. Conservation laws have also been used for existence, uniqueness and stability analysis of PDE systems. Mathematically, conservation laws can be systematically calculated, both for variational problems (Noether's theorem) and for non-variational problems $[18,19]$.

In the current paper, we consider another application of conservation laws. For any given PDE system, one can systematically construct a set (tree) of PDE systems nonlocally related to it $[20,21]$ arising from admitted conservation laws. All systems within such a tree have the same solution set as the given system. The analysis of a given PDE model through consideration of PDE systems nonlocally related to it, using any general method, can lead to new results, since the systems within a tree are related in a nonlocal manner.

We study the complete set of equations of nonlinear elasticity in a dynamical context, considering both their Lagrangian and Eulerian formulations. In Sect. 2, we derive the system of dynamic compressible nonlinear elasticity equations in the Lagrangian framework. We then focus on the one-dimensional situation and obtain the corresponding Euler and Lagrange PDE systems. In Sect.3, we find and use local conservation laws of the Euler system to construct nonlocally related systems of equations involving potential variables (potential systems). In particular, we discover an important direct connection between the systems formulated in Lagrangian and Eulerian coordinates: the Lagrange system arises as a potential system of the Euler system. In Sect. 4, classifications of admitted point symmetries in terms of constitutive functions are presented for both the Eulerian and Lagrangian formulations. Here it is shown that for both the Euler and Lagrange PDE systems, there exist cases where a symmetry is local for one system and nonlocal for the other. New group invariant solutions, arising from a nonlocal symmetry of the Euler system in a physically interesting case, are presented in Sect. 5. Some further perspectives are discussed in Sect.6.

\section{Nonlinear elasticity: the boundary-value problem}

The central problem in nonlinear elasticity consists in finding the displacement of the material points of an elastic body undergoing strains and stresses under the action of external forces, including body forces and applied tractions. In a BVP, the nonlinearity arises from the kinematics, giving rise to the so-called geometrical nonlinearity, and/or from the constitutive law, relating stresses to strains. This second source of nonlinearity is called material nonlinearity. The framework of nonlinear elasticity is suitable for materials undergoing large deformations (strains), such as elastomers and certain classes of polymers, which are capable of sustaining strains as large as a few hundred per cent without rupturing. 
In order to set the stage, first recall the underlying kinematics. At time $t_{0}$, the solid body occupies the spatial region $\bar{\Omega}_{0} \in \mathbb{R}^{3}$ (reference, or Lagrange, configuration). Here $\Omega_{0}$ is an open bounded connected subset of $\mathbb{R}^{3}$ having a Lipschitz boundary [22]. When loaded by volumetric forces $\mathbf{f}_{0}^{\prime}(\mathbf{y}, t)$ and surface tractions $\mathbf{t}_{0}$ (acting on the boundary $\partial \bar{\Omega}_{0}$ ), material points labeled by their initial positions $\mathbf{y}$ in the reference configuration undergo a motion to a deformed configuration $\bar{\Omega}$, given by the point mapping $\phi: \bar{\Omega}_{0} \times \mathbb{R} \rightarrow \bar{\Omega}$. The actual position $\mathbf{x}$ of a material point labeled by $\mathbf{y}$ at time $t$ is given by

$\mathbf{x}=\boldsymbol{\phi}(\mathbf{y}, t)$.

The set of points $\phi(\mathbf{y}, t)$ in $\mathbb{R}^{3}$ defines the deformed configuration, also called the Eulerian configuration [22, 23]. The parametrization of the material points can be done either in Lagrangian coordinates $(\mathbf{y}, t)$ or in terms of Eulerian coordinates $(\mathbf{x}, t)$.

The mapping $\boldsymbol{\phi}$ must be sufficiently smooth (the regularity conditions depending on the particular problem) and satisfy the orientation preserving condition

$J=\operatorname{det}(\nabla \boldsymbol{\phi}(\mathbf{y}, t))>0$,

where the deformation gradient $\mathbf{F}=\nabla \boldsymbol{\phi}(\mathbf{y}, t)$ is an invertible matrix (second order tensor) with components $F_{i j}=$ $\partial \phi_{i} / \partial y_{j}$, and $J$ is the Jacobian of the mapping $\boldsymbol{\phi}(\mathbf{y}, t)$. The deformation gradient is the mapping between the tangent spaces at the points $\mathbf{y}$ and $\mathbf{x}$, which locally approximates the point motion $\boldsymbol{\phi}$ from $\bar{\Omega}_{0}$ to $\bar{\Omega}$. For simple materials in the sense of Noll [24], this gives a sufficiently accurate characterization of the kinematics. The Jacobian is given by $J=\rho_{0} / \rho$, where $\rho_{0}=\rho_{0}(\mathbf{y})=\rho\left(\mathbf{x}, t_{0}\right)$ is the initial mass density at a material point, and $\rho=\rho(\mathbf{x}, t)$ is the actual density at time $t$. The velocity of the material point having the Eulerian coordinates $(\mathbf{x}, t)$ is given by $\mathbf{v}(\mathbf{x}, t)=\partial \mathbf{x} / \partial t$.

The volumetric body forces $\mathbf{f}_{0}^{\prime}(\mathbf{y}, t)$ in the Lagrangian framework have the Eulerian counterparts $\mathbf{f}^{\prime}(\mathbf{x}, t)$. The associated body forces per unit mass $\mathbf{f}_{0}(\mathbf{y}, t)$ and $\mathbf{f}(\mathbf{x}, t)$ satisfy

$\mathbf{f}_{0}^{\prime}(\mathbf{y}, t)=\rho_{0}(\mathbf{y}) \mathbf{f}_{0}(\mathbf{y}, t), \quad \mathbf{f}^{\prime}(\mathbf{x}, t)=\rho(\mathbf{x}, t) \mathbf{f}(\mathbf{x}, t)$.

The work performed by the volumetric forces is observer-invariant [22], i.e. $\mathbf{f}^{\prime}(\mathbf{x}, t) \cdot \mathrm{d} \mathbf{x}=\mathbf{f}_{0}^{\prime}(\mathbf{y}, t) \cdot \mathrm{d} \mathbf{y}$, so that $\mathbf{f}_{0}^{\prime}(\mathbf{y}, t)=J \mathbf{f}^{\prime}(\mathbf{x}, t)$. Consequently,

$\mathbf{f}_{0}(\mathbf{y}, t)=\mathbf{f}(\mathbf{x}, t)$

for the body forces per unit mass in both Lagrangian and Eulerian frameworks [22]. The BVP of nonlinear elasticity is formulated below in both the Lagrangian and Eulerian frameworks.

\subsection{BVP in the Lagrangian framework}

A hyperelastic or Green elastic material is an ideal elastic material for which the stress-strain relationship follows from a strain-energy density function [23]. It is the material model most suited to the analysis of elastomers. In general, the response of an elastic material is given in terms of the first Piola-Kirchhoff stress by $\mathbf{T}=\mathbf{T}(\mathbf{y}, \mathbf{F})$. A hyperelastic material assumes the existence of a scalar-valued volumetric strain-energy function $W=W(\mathbf{y}, \mathbf{F})$ in the reference configuration, encapsulating all information regarding the material behavior, and $\mathbf{T}$ is defined by

$\mathbf{T}(\mathbf{y}, \mathbf{F})=\frac{\partial W(\mathbf{y}, \mathbf{F})}{\partial \mathbf{F}}$.

For one-dimensional, longitudinal motions, the longitudinal component of Piola-Kirchhoff stress is a scalar-valued function given by

$T(y, q)=\frac{\partial W(y, q)}{\partial q}$,

where $F=q=\rho_{0} / \rho$. 
The 3D Lagrangian formulation of the BVP of nonlinear elasticity is then given by

$J=\operatorname{det}(\mathbf{F})=\frac{\rho_{0}}{\rho}, \quad \mathbf{v}=\mathbf{x}_{t}, \quad \rho_{0} \mathbf{v}_{t}=\operatorname{Div} \mathbf{T}+\mathbf{f}_{0}^{\prime}(\mathbf{y}, t)$,

$\mathbf{F} \cdot \mathbf{T}^{t}=\mathbf{T} \cdot \mathbf{F}^{t}, \quad$ with $\mathbf{T}(\mathbf{y}, \mathbf{F})=\frac{\partial W(\mathbf{y}, \mathbf{F})}{\partial \mathbf{F}}$,

where the operator "Div" denotes the material divergence in terms of Lagrangian coordinates, a superscript " $t$ " denotes the transpose, and $W(\mathbf{y}, \mathbf{F})$ is a constitutive function corresponding to a specific material behavior. In (2.5) and below, roman subscripts denote partial derivatives.

The first equation in (2.5) represents mass conservation, the second defines the velocity field, and the third equation expresses the conservation of momentum, with the precise form of the material response, i.e., the relation $\mathbf{T}=\mathbf{T}(\mathbf{y}, \mathbf{F})$, requiring the specification of the constitutive law as additional information. The stress tensor is not symmetric (contrary to the situation in the Eulerian description), but nevertheless satisfies some symmetry condition with respect to the tangent mapping $\mathbf{F}$ (the fourth equation in (2.5)), which restricts the number of unknown components of $\mathbf{T}$ to six.

The equations in (2.5) have to be complemented by traction boundary conditions satisfied by the stress tensor:

$\mathbf{T} \cdot \mathbf{N}=\mathbf{t}_{0}$

on the portion of the boundary $\partial \bar{\Omega}_{0}$ having the exterior normal $\mathbf{N}$, with $\mathbf{t}_{0}$ a given vector. Initial conditions for the velocity have further to be specified, together with boundary conditions for the traction, so that the system (2.5) defines a well-posed BVP.

Using the force relations (2.1), (2.2), we find that in the one-dimensional case, $f_{0}^{\prime}(y, t)=\rho_{0} f(x, t)$, and hence the one-dimensional Lagrange system is given by

$q=x_{y}, \quad v=x_{t}, \quad \rho_{0} v_{t}=T_{y}+\rho_{0} f(x, t), \quad T=K_{1}(y, q)$,

where $q, v, x$ and $T$ are the dependent variables depending on $(y, t)$, and $K_{1}(y, q)=\partial W(y, q) / \partial q$ is a materialdependent constitutive function [22]. For the rest of this paper, we will only consider homogeneous materials for which $K_{1}(y, q) \equiv K_{1}(q)$.

\subsection{BVP in the Eulerian framework}

The Cauchy stress tensor is given by

$\sigma=J^{-1} \mathbf{T} \cdot \mathbf{F}^{t}$.

For homogeneous isotropic materials, the axiom of material frame indifference allows one to express $\sigma$ in terms of the left Cauchy-Green strain tensor $\mathbf{B}=\mathbf{F} \cdot \mathbf{F}^{t}$ ([22], Ch. 3):

$\sigma=\phi_{0}\left(\mathbf{i}_{\mathbf{B}}\right) \mathbf{I}+\phi_{1}\left(\mathbf{i}_{\mathbf{B}}\right) \mathbf{B}+\phi_{2}\left(\mathbf{i}_{\mathbf{B}}\right) \mathbf{B}^{2}$,

where $\phi_{0}, \phi_{1}, \phi_{2}$ are functions of the principal invariants $\mathbf{i}_{\mathbf{B}}=\left(i_{1}, i_{2}, i_{3}\right)$ of $\mathbf{B}[22,24,25]$.

The BVP satisfied by the dependent variables $(\rho, \mathbf{v}, \boldsymbol{\sigma})$ in terms of the spatial and temporal coordinates $(\mathbf{x}, t)$ is now given by the Euler system:

$\rho_{t}+\operatorname{div}(\rho \mathbf{v})=0, \quad \operatorname{div} \sigma+\rho \mathbf{f}(\mathbf{x}, t)=\rho\left(\mathbf{v}_{t}+(\mathbf{v} \cdot \nabla) \mathbf{v}\right)$,

$\sigma=\sigma^{t}, \quad \sigma=\phi_{0}\left(\mathbf{i}_{\mathbf{B}}\right) \mathbf{I}+\phi_{1}\left(\mathbf{i}_{\mathbf{B}}\right) \mathbf{B}+\phi_{2}\left(\mathbf{i}_{\mathbf{B}}\right) \mathbf{B}^{2}$,

where the operator "div" denotes spatial divergence. The equations in (2.9) respectively represent mass conservation, conservation of momentum (dynamical equilibrium), the symmetry of the Cauchy stress tensor (which consequently has six independent unknown components), and the material's constitutive law. The equations in (2.9) have to be complemented by boundary conditions for the velocity $\mathbf{v}$ and traction boundary conditions satisfied by the stress:

$\boldsymbol{\sigma} \cdot \mathbf{n}=\mathbf{t}(\mathbf{x})$

on a portion of the boundary $\partial \bar{\Omega}$ having the exterior normal $\mathbf{n}$. Initial conditions for the variables $(\rho, \mathbf{v})$ also need to be specified. 
2.3 Field equations in the one-dimensional case

In the one-dimensional situation, the Cauchy-Green strain tensor $\mathbf{B}$ is a scalar: $\mathbf{B}=F^{2}$. Since $F=q=\rho_{0} / \rho$, for the Cauchy stress we have $\sigma=\sigma(\rho)$. Consequently, the one-dimensional analog of the Euler system (2.9) is given by

$\mathbf{E}\{x, t ; v, \sigma, \rho\}:\left\{\begin{array}{l}\rho_{t}+(\rho v)_{x}=0, \\ \sigma_{x}+\rho f(x, t)=\rho\left(v_{t}+v v_{x}\right), \\ \sigma=K(\rho) .\end{array}\right.$

[For the rest of this paper we only consider conservative forces $f(x, t) \equiv f(x)$.] Here the independent variables are $(x, t)$, the dependent variables are $(\rho, v, \sigma)$, and some freedom of choice is given by the possible forms of $f=f(x)$ and the constitutive function $K=K(\rho)$, describing the load per unit mass and the constitutive response of the material, respectively.

For the rest of the paper, we work in dimensionless variables. ${ }^{1}$

From definition (2.7), it follows that $\sigma=T$. Therefore the 1D Lagrangian system (2.6) in dimensionless variables simplifies to

$\mathbf{L}\{y, t ; v, \sigma, q, x\}: \begin{cases}v=x_{t}, & \sigma=K(1 / q), \\ q=x_{y}, & v_{t}=\sigma_{y}+f(x) .\end{cases}$

In the Lagrangian system, the independent variables are $(y, t)$, the dependent variables are $(x, v, q, \sigma)$, and the free functions are $f=f(x)$ and $K_{1}(q)=K(1 / q)$.

Remark 1 In the case $f(x)=0$ (or, more generally, in the equivalent case $f(x)=$ const, see (4.6) below), the Euler system (2.10) is linearizable by a hodograph transformation, since it is a quasilinear system with coefficients depending only on independent variables (see e.g. [4]).

Remark 2 Upon the substitution $\sigma=-p$, both the Euler system (2.10) and the Lagrange system (2.11) coincide with forced isentropic Euler equations of inviscid fluid motion, in Eulerian and Lagrangian coordinates respectively. Nonlocally related PDE systems for non-forced 1D fluid dynamics equations with a more general constitutive function were studied in $[20,21]$.

\section{Nonlocally related systems of nonlinear elasticity}

\subsection{PDE systems nonlocally related to a given one}

Consider two PDE systems $\mathbf{A}\{\mathbf{x} ; \mathbf{u}\}=0$ and $\mathbf{B}\{\mathbf{x} ; \mathbf{u}, \mathbf{v}\}=0$ with independent variables $\mathbf{x}$ and dependent variables $\mathbf{u}, \mathbf{v}$. Systems $\mathbf{A}$ and $\mathbf{B}$ are nonlocally related if

1. System $\mathbf{B}$ includes additional dependent variable(s) $\mathbf{v}$ only in the form of derivatives.

2. Exclusion of the dependent variable(s) $\mathbf{v}$ from $\mathbf{B}$, through compatibility relations (e.g. $v_{x t}=v_{t x}$ ), yields the system A.

Nonlocally related PDE systems are equivalent in the sense that the solution set of one such system can be found from the solution set of the other. At the same time, the analysis of a system of PDEs through consideration of nonlocally related systems can be of great value. In particular, for a given system of PDEs, through Lie's algorithm

$\overline{{ }^{1} \text { We choose }} x=x_{1} \widetilde{x}, t=t_{1} \widetilde{t}, v(x, t)=\frac{x_{1}}{t_{1}} \widetilde{v}(\widetilde{x}, \widetilde{t}), \rho(x, t)=\rho_{0} \widetilde{\rho}(\widetilde{x}, \widetilde{t}), \sigma(x, t)=K(\rho(x, t))=\frac{x_{1}^{2} \rho_{0}}{t_{1}^{2}} \widetilde{K}(\widetilde{\rho}(\widetilde{x}, \widetilde{t})), f(x)=\frac{x_{1}}{t_{1}^{2}} \widetilde{f}(\widetilde{x})$, $\widetilde{q}=q=1 / \widetilde{\rho}$. Here $x_{1}, t_{1}, \rho_{0}=$ const. The Euler system (2.10) in dimensionless variables $\widetilde{x}, \widetilde{t}, \widetilde{\rho}, \widetilde{v}, \widetilde{\sigma}$ has exactly the same form as in original variables. Then for dimensionless variables, we omit tildas. The same is done for all other PDE systems below. In particular, for the Lagrange coordinate, $y=x_{1} \tilde{y}$. 
applied to a nonlocally related system, one can systematically calculate nonlocal symmetries (which in turn are useful for obtaining new exact solutions from known ones), construct (further) invariant and nonclassical solutions, as well as obtain linearizations, etc. (Examples are found in $[20,21,26,27]$.) Perhaps more importantly, since each such related system contains all solutions of the given system, any general method of analysis (qualitative, numerical, perturbation, conservation laws, etc.) considered for a given PDE system may be tried again on any PDE system nonlocally related to it. In this way, new results may be obtained for any method of analysis that is not coordinate-dependent since the systems are related in a nonlocal manner.

Most importantly, for any given system of PDEs, one can systematically construct an extended tree of nonlocally related potential systems and subsystems [20,21].

Let $\mathbf{G}$ be a system of $m$ partial differential equations

$\mathbf{G}\{\mathbf{x} ; \mathbf{u}\}=0: \quad G_{1}\{\mathbf{x} ; \mathbf{u}\}=0 \quad \ldots, \quad G_{m}\{\mathbf{x} ; \mathbf{u}\}=0$

with $M$ independent variables $\mathbf{x}=\left(x^{1}, \ldots, x^{M}\right)$ and $N$ dependent variables $\mathbf{u}=\left(u^{1}, \ldots, u^{N}\right)$. Let $\partial^{l} \mathbf{u}$ denote the set of all partial derivatives of $\mathbf{u}$ of order $l$.

Each conservation law of system (3.1),

$D_{i} \Phi^{i}\left(\mathbf{x}, \mathbf{u}, \partial \mathbf{u}, \ldots, \partial^{r} \mathbf{u}\right)=0$,

gives rise to one or more potential variables and a corresponding nonlocally related potential system. (The summation in a repeated index is assumed in (3.2) and throughout the paper.) In particular, for systems with $M=2$ independent variables $(x, t)$, each conservation law

$D_{x} X\left(x, t, \mathbf{u}, \partial \mathbf{u}, \ldots, \partial^{r} \mathbf{u}\right)+D_{t} T\left(x, t, \mathbf{u}, \partial \mathbf{u}, \ldots, \partial^{r} \mathbf{u}\right)=0$,

where $D_{x}$ and $D_{t}$ denote total derivatives with respect to $x$ and $t$, respectively, yields a pair of potential equations of the form

$\mathcal{P}: w_{x}=T\left(x, t, \mathbf{u}, \partial \mathbf{u}, \ldots, \partial^{r} \mathbf{u}\right), \quad w_{t}=-X\left(x, t, \mathbf{u}, \partial \mathbf{u}, \ldots, \partial^{r} \mathbf{u}\right)$.

For each conservation law (3.3), corresponding potential equations (3.4) can be appended to the given system (3.1) to yield a potential system $\mathbf{G}_{\mathbf{P}}$ :

$\mathbf{G}_{\mathbf{P}}\{x, t ; \mathbf{u}, w\}:\left\{\begin{array}{l}w_{x}=T\left(x, t, \mathbf{u}, \partial \mathbf{u}, \ldots, \partial^{r} \mathbf{u}\right), \\ w_{t}=-X\left(x, t, \mathbf{u}, \partial \mathbf{u}, \ldots, \partial^{r} \mathbf{u}\right), \\ G_{1}\{x, t ; \mathbf{u}\}=0, \ldots, \quad G_{m}\{x, t ; \mathbf{u}\}=0 .\end{array}\right.$

Subsystems are obtained by excluding one or more dependent variables from the given system or potential systems, including subsystems obtained after an interchange of dependent and independent variables. Moreover, a subsystem $\mathbf{F}$ of a PDE system $\mathbf{F}$ is nonlocally related to $\mathbf{F}$ if it is obtained by excluding from $\mathbf{F}$ a dependent variable $u$ that is present in $\mathbf{F}$ only in terms of derivatives of $u$.

For a discussion of nonlocally related PDE systems for $M \geq 2$ independent variables, see [4, 20, 21].

Below we use obvious conservation laws of the 1D system of nonlinear elasticity derived in Sect. 2.3 to construct a tree of nonlocally related PDE systems. The systems of the tree are used further to classify local and nonlocal symmetries and derive consequent invariant solutions of PDE systems for nonlinear elasticity.

\subsection{Nonlocally related systems of one-dimensional nonlinear elasticity equations}

We now consider nonlocally related systems for both the Euler system $\mathbf{E}$ (2.10) and the Lagrange system $\mathbf{L}$ (2.11) for $1 \mathrm{D}$ nonlinear elasticity equations. Moreover, we show a systematic connection between these systems through the framework discussed in Sect.3.1. 


\subsubsection{Nonlocal relation between Euler and Lagrange systems}

The first equation of the Euler system $\mathbf{E}$ (2.10) is in the form of a conservation law (mass conservation) as it stands. Hence a potential $w$ can be introduced. The corresponding potential system takes the form

$\mathbf{E W}\{x, t ; v, \sigma, \rho, w\}: \begin{cases}w_{t}=-\rho v, & \sigma=K(\rho), \\ w_{x}=\rho, & \sigma_{x}+\rho f(x)=\rho\left(v_{t}+v v_{x}\right) .\end{cases}$

It is remarkable that a local 1:1 point transformation (an interchange of a dependent and independent variable) of the system $\mathbf{E W}$ with $w=y$ and $t$ treated as independent variables, and $x, v, \sigma, q=1 / \rho$ as dependent variables, directly yields the Lagrange system $\mathbf{L}$ given by (2.11). The same situation takes place for the equations of planar gas dynamics in Eulerian and Lagrangian coordinates [20]. Hence the systems $\mathbf{E W}$ and $\mathbf{L}$ are locally related to each other, but nonlocally related to the Euler system $\mathbf{E}$.

In the Lagrange system $\mathbf{L}$ (or equivalently $\mathbf{E W}$ ), the variable with $y=w=\int \rho(x, t) \mathrm{d} x$ is a mass coordinate.

\subsubsection{Subsystems of Euler and Lagrange systems}

Excluding the dependent variable $\sigma$ from the Euler system $\mathbf{E}$, we obtain a locally related subsystem:

$\rho_{t}+(\rho v)_{x}=0, \quad K^{\prime}(\rho) \rho_{x}=\rho\left[v_{t}+v v_{x}-f(x)\right]$.

From the first two equations of the Lagrange system $\mathbf{L}$ (2.11), one can exclude dependent variables $q$ and $v$ to obtain a locally related subsystem

$x_{t t}=-\frac{1}{x_{y}^{2}} K^{\prime}\left(\frac{1}{x_{y}}\right) x_{y y}+f(x)$.

It is easy to see that, when $K(\rho)=A \rho^{-1}, A=$ const, the subsystem (3.8) becomes quasilinear, and linear if $f(x)$ is a linear function. This situation does not follow from a direct consideration of the Euler system $\mathbf{E}$.

Since subsystems (3.7) and (3.8) are locally related subsystems of systems $\mathbf{E}$ and $\mathbf{L}$, respectively, they will not be further considered.

\subsubsection{General conservation laws and further nonlocally related systems}

To further extend the tree of nonlocally related systems of one-dimensional nonlinear elasticity equations, one should find additional conservation laws and consider potential systems of PDE systems $\mathbf{E}, \mathbf{E W}$ or $\mathbf{L}$. In particular, for the Euler system $\mathbf{E}$, we observe that the equation of conservation of momentum i.e., the second equation of (2.10), can be written in the divergence form

$D_{t}(v-f(x) t)+D_{x}\left(\frac{v^{2}}{2}-M(\rho)\right)=0$,

where $M(\rho)=\int \frac{K^{\prime}(\rho)}{\rho} \mathrm{d} \rho$. Introducing a potential variable $r(x, t)$, we obtain potential equations

$r_{x}=v-f(x) t, \quad r_{t}=M(\rho)-\frac{v^{2}}{2}$.

For the Euler system E, there also exists another conservation law (conservation of energy)

$D_{t}\left(\rho \frac{v^{2}}{2}-\int M(\rho) \mathrm{d} \rho-\rho \int f(x) \mathrm{d} x\right)+D_{x}\left(\rho v\left[\frac{v^{2}}{2}-M(\rho)-\int f(x) \mathrm{d} x\right]\right)=0$.

This conservation law yields potential equations

$s_{x}=\rho \frac{v^{2}}{2}-\int M(\rho) \mathrm{d} \rho-\rho \int f(x) \mathrm{d} x, \quad s_{t}=-\rho v\left[\frac{v^{2}}{2}-M(\rho)-\int f(x) \mathrm{d} x\right]$. 
Remark 3 The nonlocal variable $s(x, t)=\int\left(\rho \frac{v^{2}}{2}-\int M(\rho) \mathrm{d} \rho-\rho \int f(x) \mathrm{d} x\right) \mathrm{d} x$ is an "energy coordinate", analogous to the mass coordinate $w$ and the "velocity coordinate" $r$.

In summary, the three conservation laws (mass, the one related to momentum and energy) yield the following seven distinct nonlocally related (potential) systems of the Euler system $\mathbf{E}$ [21].

- Three singlet potential systems: EW (3.6);

$\mathbf{E R}\{x, t ; v, \sigma, \rho, r\}:\left\{\begin{array}{l}\rho_{t}+(\rho v)_{x}=0, r_{t}=M(\rho)-v^{2} / 2, \\ r_{x}=v-f(x) t, \sigma=K(\rho) ;\end{array}\right.$

and

$\mathbf{E S}\{x, t ; v, \sigma, \rho, s\}:\left\{\begin{array}{l}\rho_{t}+(\rho v)_{x}=0, \\ s_{x}=\rho v^{2} / 2-\int M(\rho) \mathrm{d} \rho-\rho \int f(x) \mathrm{d} x, \\ s_{t}=-\rho v\left[v^{2} / 2-M(\rho)-\int f(x) \mathrm{d} x\right], \\ \sigma_{x}+\rho f(x)=\rho\left(v_{t}+v v_{x}\right), \quad \sigma=K(\rho) .\end{array}\right.$

- Three couplet potential systems:

$\mathbf{E R W}\{x, t ; v, \sigma, \rho, r, w\}: \begin{cases}\rho_{t}+(\rho v)_{x}=0, & w_{x}=\rho, \\ r_{t}=M(\rho)-v^{2} / 2, & \sigma=K(\rho), \\ r_{x}=v-f(x) t, & w_{t}=-\rho v ;\end{cases}$

$\operatorname{ESW}\{x, t ; v, \sigma, \rho, s, w\}:\left\{\begin{array}{l}\rho_{t}+(\rho v)_{x}=0, \\ s_{x}=\rho v^{2} / 2-\int M(\rho) \mathrm{d} \rho-\rho \int f(x) \mathrm{d} x, \\ s_{t}=-\rho v\left[v^{2} / 2-M(\rho)-\int f(x) \mathrm{d} x\right], \\ w_{t}=-\rho v, \quad \sigma=K(\rho), \\ w_{x}=\rho, \quad \sigma_{x}+\rho f(x)=\rho\left(v_{t}+v v_{x}\right) ;\end{array}\right.$

and

$\boldsymbol{E R S}\{x, t ; v, \sigma, \rho, r, s\}:\left\{\begin{array}{l}\rho_{t}+(\rho v)_{x}=0, \\ r_{x}=v-f(x) t, \quad r_{t}=M(\rho)-v^{2} / 2, \\ s_{x}=\rho v^{2} / 2-\int M(\rho) \mathrm{d} \rho-\rho \int f(x) \mathrm{d} x, \\ s_{t}=-\rho v\left[v^{2} / 2-M(\rho)-\int f(x) \mathrm{d} x\right], \\ \sigma_{x}+\rho f(x)=\rho\left(v_{t}+v v_{x}\right), \quad \sigma=K(\rho) .\end{array}\right.$

- One triplet potential system

$\operatorname{ERSW}\{x, t ; v, \sigma, \rho, r, s, w\}:\left\{\begin{array}{l}\rho_{t}+(\rho v)_{x}=0, \\ r_{x}=v-f(x) t, \quad r_{t}=M(\rho)-v^{2} / 2, \\ s_{x}=\rho v^{2} / 2-\int M(\rho) \mathrm{d} \rho-\rho \int f(x) \mathrm{d} x, \\ s_{t}=-\rho v\left[v^{2} / 2-M(\rho)-\int f(x) \mathrm{d} x\right], \\ w_{x}=\rho, \quad w_{t}=-\rho v, \\ \sigma_{x}+\rho f(x)=\rho\left(v_{t}+v v_{x}\right), \quad \sigma=K(\rho) .\end{array}\right.$ 
Fig. 1 The tree of nonlocally related systems of nonlinear elasticity. The dotted box corresponds to nonlocally related systems that arise for the case $f(x)=$ const

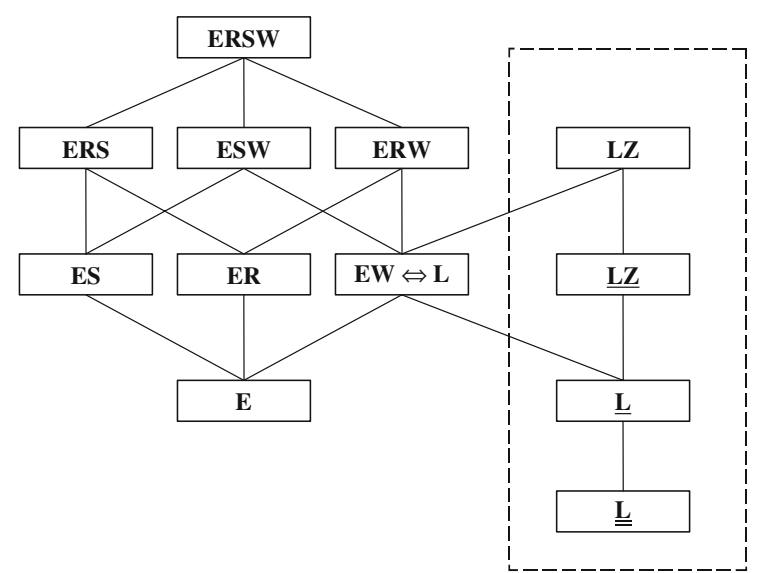

Thus we have found that for arbitrary forms of constitutive functions $K(\rho)$ and $f(x)$, one has a tree of equivalent and nonlocally related systems of nonlinear elasticity, consisting of eight $P D E$ systems $\mathbf{E}, \mathbf{L} \Leftrightarrow \mathbf{E W}, \mathbf{E R}, \mathbf{E S}$, ERW, ESW, ERS, and ERSW. This tree is shown in Fig. 1.

\subsubsection{Particular tree extensions}

For the case $f(x)=f_{1}=$ const, further nonlocally related systems arise from the Lagrange system $\mathbf{L}$ (2.11). [As we will see below in Sect. 4.2.1, the case $f(x)=$ const is equivalent to the case $f(x)=0$.] In particular, excluding $x$ from (2.11) yields a nonlocally related subsystem

$\underline{\mathbf{L}}\{y, t ; v, q, \sigma\}: \quad q_{t}=v_{y}, \quad v_{t}=\sigma_{y}+f_{1}, \quad \sigma=K(1 / q)$.

Furthermore, excluding $v$ from (3.19) by cross-differentiation and substituting for $\sigma$ yields another nonlocally related subsystem given by

$\underline{\underline{\mathbf{L}}}\{y, t ; q\}: \quad q_{t t}=(K(1 / q))_{y y}$.

Moreover, when $f(x)=$ const, the third equation of $\mathbf{L}$ can be used to introduce a potential variable $z$ and the consequent nonlocally related potential system

$\mathbf{L Z}\{y, t ; v, \sigma, q, x, z\}:\left\{\begin{array}{l}q=x_{y}, \quad v=x_{t}, \quad z_{y}=v, \\ z_{t}=\sigma+f_{1} y, \quad \sigma=K(1 / q) .\end{array}\right.$

The potential system $\mathbf{L Z}$ has a nonlocally related subsystem, obtained by excluding $x$, given by

$\underline{\mathbf{L Z}}\{y, t ; v, \sigma, q, z\}:\left\{\begin{array}{cc}q_{t}=v_{y}, & z_{t}=\sigma+f_{1} y, \\ z_{y}=v, & \sigma=K(1 / q) .\end{array}\right.$

The tree for the above listed nonlocally related systems and subsystems is presented in Fig. 1.

\section{Point and nonlocal symmetries of nonlinear elasticity equations}

4.1 Point and nonlocal symmetries of differential equations

A symmetry of a system of PDEs (3.1) is any transformation of its solution manifold into itself (i.e., a symmetry transforms any solution to another solution of the same system).

Lie's algorithm is used to find one-parameter $(\varepsilon)$ Lie groups of point transformations (point symmetries)

$\left(x^{*}\right)^{i}=f^{i}(\mathbf{x}, \mathbf{u} ; \varepsilon), \quad i=1, \ldots, M ; \quad\left(u^{*}\right)^{j}=g^{j}(\mathbf{x}, \mathbf{u} ; \varepsilon), \quad j=1, \ldots, N$,

admitted by a given PDE system (3.1) [1-4] such that $\mathbf{G}\left\{\mathbf{x}^{*}, \mathbf{u}^{*}\right\}=0$ if and only if $\mathbf{G}\{\mathbf{x}, \mathbf{u}\}=0$. 
The global Lie transformation group (4.1) is in one-to-one correspondence with local transformations

$\left(x^{*}\right)^{i}=x^{i}+\varepsilon \xi^{i}(\mathbf{x}, \mathbf{u})+O\left(\varepsilon^{2}\right), \quad i=1, \ldots, M$,

$\left(u^{*}\right)^{j}=u^{j}+\varepsilon \eta^{j}(\mathbf{x}, \mathbf{u})+O\left(\varepsilon^{2}\right), \quad j=1, \ldots, N$,

where $\xi^{i}, \eta^{j}$ are components of a vector field (infinitesimal generator)

$X=\xi^{i}(\mathbf{x}, \mathbf{u}) \frac{\partial}{\partial x^{i}}+\eta^{j}(\mathbf{x}, \mathbf{u}) \frac{\partial}{\partial u^{j}}$

tangent to the solution manifold of the given system (3.1). There exist various software packages to find the infinitesimal generators (4.3) of point symmetries admitted by a given system of differential equations (e.g. [28, 29]). In this work we used [28].

In general, symmetry transformations are defined topologically and are not restricted to point or, more generally, local transformations acting on the given system's dependent and independent variables and derivatives of dependent variables. In particular, nonlocal symmetries can be admitted by systems of differential equations.

For a given system $\mathbf{G}$ (3.1), nonlocal symmetries called potential symmetries can arise naturally by applying Lie's algorithm to a related potential system $\mathbf{G}_{\mathbf{P}}$ (3.5). In particular, suppose $\mathbf{G}_{\mathbf{P}}$ admits a point symmetry

$$
\begin{array}{ll}
x^{*}=x+\varepsilon \xi \mathbf{P}(x, t, \mathbf{u}, w)+O\left(\varepsilon^{2}\right), & t^{*}=t+\varepsilon \tau_{\mathbf{P}}(x, t, \mathbf{u}, w)+O\left(\varepsilon^{2}\right), \\
u^{* j}=u^{j}+\varepsilon \eta^{j} \mathbf{P}(x, t, \mathbf{u}, w)+O\left(\varepsilon^{2}\right), & w^{*}=w+\varepsilon \zeta_{\mathbf{P}}(x, t, \mathbf{u}, w)+O\left(\varepsilon^{2}\right)
\end{array}
$$

with infinitesimal generator

$X=\xi_{\mathbf{P}}(x, t, \mathbf{u}, w) \frac{\partial}{\partial x}+\tau_{\mathbf{P}}(x, t, \mathbf{u}, w) \frac{\partial}{\partial t}+\eta_{\mathbf{P}}^{j}(x, t, \mathbf{u}, w) \frac{\partial}{\partial u^{j}}+\zeta_{\mathbf{P}}(x, t, \mathbf{u}, w) \frac{\partial}{\partial w}$.

A point symmetry (4.4) of the potential system $\mathbf{G}_{\mathbf{P}}$ is a potential symmetry of the given system $\mathbf{G}$ if and only if $\left(\frac{\partial \xi \mathbf{P}}{\partial w}\right)^{2}+\left(\frac{\partial \tau \mathbf{P}}{\partial w}\right)^{2}+\sum_{j=1}^{n}\left(\frac{\partial \eta_{\mathbf{P}}^{j}}{\partial w}\right)^{2}>0$, i.e., the infinitesimals $\xi_{\mathbf{P}}, \tau_{\mathbf{P}}, \eta_{\mathbf{P}}^{j}$ essentially depend on the potential variable $w$. Consequently, a potential symmetry is a nonlocal symmetry of the given system (3.1) [4].

Remark 4 Nonlocal symmetries of a given system can also arise as point symmetries of its nonlocally related subsystems (see [20].) Thus a comprehensive symmetry analysis of a given PDE system includes the study of point symmetries of its nonlocally related potential systems and subsystems.

\subsection{Point and nonlocal symmetry classification of nonlinear elasticity equations}

In this section, we classify and compare symmetries of some of the nonlocally related systems of PDEs for nonlinear elasticity introduced in Sects. 2 and 3. In particular, we present the complete point-symmetry classifications for both the Euler system $\mathbf{E}$ (2.10) and the potential Euler (Lagrange) system EW (3.6), and compute symmetries admitted by the potential system $\mathbf{E R}$ which are nonlocal symmetries of $\mathbf{E}$ and/or $\mathbf{E W}$.

The case $K(\rho)=0$ is not realistic and therefore is not considered. We also do not compute symmetries in the degenerate case of constant stress $\sigma=K(\rho)=$ const, and in the linearizable case $f(x)=$ const (see Remark 1 ).

\subsubsection{Point-symmetry classification of the Euler system $\mathbf{E}$ (2.10)}

The Euler system $\mathbf{E}$ (2.10) is invariant under a seven-parameter group of equivalence transformations

$x=a_{1} x_{1}+a_{2} t_{1}+a_{3} t_{1}^{2}+a_{4}, \quad t=a_{5} t_{1}+a_{6}$,

$\rho=a_{7} \rho_{1}, \quad v=\frac{a_{1}}{a_{5}} v_{1}+\frac{2 a_{3}}{a_{5}^{2}} t_{1}+\frac{a_{2}}{a_{5}}$,

$f(x)=\frac{a_{1}}{a_{5}^{2}} f_{1}\left(x_{1}\right)+\frac{2 a_{3}}{a_{5}^{2}}, \quad K(\rho)=\frac{a_{1}^{2} a_{7}}{a_{5}^{2}} K_{1}\left(\rho_{1}\right)$

for arbitrary constants $a_{1}, \ldots, a_{7} ; a_{1}, a_{5}, a_{7} \neq 0$.

Modulo the equivalence transformations (4.6), the point symmetries admitted by the Euler system $\mathbf{E}$ (2.10) are summarized in Table 1. 
Table 1 Point symmetries of the Euler system $\mathbf{E}$ (2.10)

\begin{tabular}{lll}
\hline$K(\rho)$ & $f(x)$ & Point symmetries \\
\hline Arbitrary & Arbitrary & $X_{1}=\frac{\partial}{\partial t}$. \\
& $1 / x$ & $X_{1}, X_{2}=x \frac{\partial}{\partial x}+t \frac{\partial}{\partial t}$. \\
& $x$ & $X_{1}, X_{3}=\mathrm{e}^{t}\left(\frac{\partial}{\partial x}+\frac{\partial}{\partial v}\right), X_{4}=\mathrm{e}^{-t}\left(\frac{\partial}{\partial x}-\frac{\partial}{\partial v}\right)$. \\
& 0 & Infinite number; linearization by a hodograph transformation. \\
& $\mathrm{e}_{1}, X_{5}=(B-1)\left(\frac{\partial}{\partial x}-\frac{1}{2} t \frac{\partial}{\partial t}+\frac{1}{2} v \frac{\partial}{\partial v}\right)+\rho \frac{\partial}{\partial \rho}$. \\
$\rho^{B}$ & $x_{1}, X_{6}=(B-1) x \frac{\partial}{\partial x}-\frac{1}{2}(d-1)(B-1) t \frac{\partial}{\partial t}+\frac{1}{2}(B-1)(d+1) v \frac{\partial}{\partial v}+(d+1) \rho \frac{\partial}{\partial \rho}$. \\
& $x^{d}$ & $X_{1}, X_{2}, X_{6}(d=-1)$. \\
& $X_{1}, X_{3}, X_{4}, X_{6}(d=1)$. \\
$\rho^{3}$ & Arbitrary & $X_{1}, X_{7}=\rho \frac{\partial}{\partial \rho}$. \\
& Arbitrary & Infinite number. \\
\hline
\end{tabular}

\subsubsection{Point-symmetry classification of the Lagrange system $\mathbf{E W} \Leftrightarrow \mathbf{L}$ (3.6)}

Since the potential Euler system EW (3.6) and the Lagrange system $\mathbf{L}$ (2.11) system are invertibly equivalent, it suffices to calculate point symmetries for only one of these systems. We perform the calculations for $\mathbf{E W}$ since it is easier to compare its symmetries with the ones admitted by the Euler system $\mathbf{E}$.

The potential Euler system $\mathbf{E W}$ (3.6) is invariant under the equivalence transformations (4.6) with $w=a_{1} a_{7} w_{1}+$ $a_{8}$, where $a_{8}$ is another arbitrary constant. Modulo these equivalence transformations, the point symmetries admitted by the Lagrange (potential Euler) system EW (3.6) are summarized in Table 2.

For arbitrary $K(\rho)$, for the case $f(x)=$ const (corresponding to $f(x)=0$ in the tables), the Euler system $\mathbf{E}$ (2.10) is linearizable by a point transformation, whereas the Lagrange system $\mathbf{E W}$ (3.6) only admits two additional symmetries $Y_{2}, Y_{3}$. (The other point symmetries of the Euler system $\mathbf{E}$ correspond to point symmetries of the Lagrange system $\mathbf{E W}$.) On the other hand, unlike the Euler system $\mathbf{E}$, the Lagrange system $\mathbf{E W}$ is linearizable by a point transformation when $K(\rho)=1 / \rho$ for $f(x)=\mathrm{e}^{x}, f(x)=x, f(x)=$ const (hence the Euler system in these cases is linearizable by a nonlocal transformation [4]).

Both the Euler system $\mathbf{E}$ and the Lagrange system $\mathbf{E W}$ admit an infinite number of point symmetries when $K(\rho)=\rho^{3}$ for an arbitrary force $f(x)$.

When $K(\rho)=\frac{1}{2}\left(\arctan \frac{1}{\rho}+\frac{\rho}{\rho^{2}+1}\right)$ or $K(\rho)=\frac{1}{4} \log \frac{\rho-1}{\rho+1}-\frac{1}{2} \frac{\rho}{\rho^{2}-1}$, for the force term $f(x)=x$, the Lagrange system $\mathbf{E W}$ (3.6) admits two additional point symmetries $\left(\left(Y_{4}, Y_{5}\right)\right.$ and $\left(Y_{6}, Y_{7}\right)$ respectively), which are nonlocal symmetries of the Euler system $\mathbf{E}$ (2.10).

When $K(\rho)=1 / \rho$, for an arbitrary force $f(x)$, the Lagrange system $\mathbf{E W}$ (3.6) admits one additional point symmetry $Y_{8}$ which is a nonlocal symmetry of the Euler system $\mathbf{E}$.

The other point symmetries of the Lagrange system $\mathbf{E W}$ correspond to point symmetries of the Euler system $\mathbf{E}$.

\subsubsection{Point-symmetry classification of the system ER (3.13)}

The "velocity-coordinate" PDE system ER (3.13) is invariant under a five-parameter group of equivalence transformations

$$
\begin{aligned}
& x=a_{1} x_{1}+a_{2}, \quad t=a_{3} t_{1}, \quad \rho=a_{4} \rho_{1}, \quad v=\frac{a_{1}}{a_{3}} v_{1}, \quad r=\frac{a_{1}^{2}}{a_{2}} r_{1}+a_{5}, \\
& f(x)=\frac{a_{1}}{a_{3}^{2}} f_{1}\left(x_{1}\right), \quad K(\rho)=\frac{a_{1}^{2} a_{4}}{a_{2}^{2}} K_{1}\left(\rho_{1}\right)
\end{aligned}
$$

for arbitrary constants $a_{1}, \ldots, a_{5} ; a_{1}, a_{3}, a_{4} \neq 0$. 
Table 2 Point symmetries of the Lagrange (potential Euler) system EW (3.6)

\begin{tabular}{|c|c|c|}
\hline$K(\rho)$ & $f(x)$ & Point symmetries \\
\hline Arbitrary & $\begin{array}{l}\text { Arbitrary } \\
1 / x \\
x \\
0\end{array}$ & $\begin{array}{l}X_{1}=\frac{\partial}{\partial t}, Y_{1}=\frac{\partial}{\partial w} \\
X_{1}, Y_{1}, X_{2}=x \frac{\partial}{\partial x}+t \frac{\partial}{\partial t}+w \frac{\partial}{\partial w} \\
X_{1}, Y_{1}, X_{3}=\mathrm{e}^{t}\left(\frac{\partial}{\partial x}+\frac{\partial}{\partial v}\right), X_{4}=\mathrm{e}^{-t}\left(\frac{\partial}{\partial x}-\frac{\partial}{\partial v}\right) \\
X_{1}, Y_{1}, Y_{2}=\frac{\partial}{\partial x}, \quad Y_{3}=\frac{\partial}{\partial v}+t \frac{\partial}{\partial x}\end{array}$ \\
\hline$\frac{1}{2} \arctan \frac{1}{\rho}+\frac{1}{2} \frac{\rho}{\rho^{2}+1}$ & $x$ & $\begin{array}{l}X_{1}, Y_{1}, Y_{4}=\frac{\mathrm{e}^{t}}{\rho}\left[\frac{\partial}{\partial t}+(v+\rho w) \frac{\partial}{\partial x}+(x+\rho w) \frac{\partial}{\partial v}-\rho\left(\rho^{2}+1\right) \frac{\partial}{\partial \rho}-\rho(x-v) \frac{\partial}{\partial w}\right] \\
Y_{5}=\frac{\mathrm{e}^{-t}}{\rho}\left[\frac{\partial}{\partial t}+(v-\rho w) \frac{\partial}{\partial x}+(x+\rho w) \frac{\partial}{\partial v}+\rho\left(\rho^{2}+1\right) \frac{\partial}{\partial \rho}+\rho(x+v) \frac{\partial}{\partial w}\right] .\end{array}$ \\
\hline$\frac{1}{4} \log \frac{\rho-1}{\rho+1}-\frac{1}{2} \frac{\rho}{\rho^{2}-1}$ & $x$ & $\begin{array}{l}X_{1}, Y_{1}, Y_{6}=\frac{\mathrm{e}^{t}}{\rho}\left[\frac{\partial}{\partial t}+(v-\rho w) \frac{\partial}{\partial x}+(x-\rho w) \frac{\partial}{\partial v}+\rho\left(\rho^{2}-1\right) \frac{\partial}{\partial \rho}-\rho(x-v) \frac{\partial}{\partial w}\right] \\
Y_{7}=\frac{\mathrm{e}^{-t}}{\rho}\left[\frac{\partial}{\partial t}+(v+\rho w) \frac{\partial}{\partial x}+(x-\rho w) \frac{\partial}{\partial v}-\rho\left(\rho^{2}-1\right) \frac{\partial}{\partial \rho}+\rho(x+v) \frac{\partial}{\partial w}\right] .\end{array}$ \\
\hline$\rho^{B}$ & $\begin{array}{l}\mathrm{e}^{x} \\
x^{d} \\
1 / x \\
x\end{array}$ & $\begin{array}{l}X_{1}, Y_{1}, X_{5}=(B-1)\left(\frac{\partial}{\partial x}-\frac{t}{2} \frac{\partial}{\partial t}+\frac{1}{2} v \frac{\partial}{\partial v}\right)+\rho \frac{\partial}{\partial \rho}+w \frac{\partial}{\partial w} . \\
X_{1}, Y_{1}, X_{6}=(B-1) x \frac{\partial}{\partial x}-\frac{1}{2}(d-1)(B-1) t \frac{\partial}{\partial t}+\frac{1}{2}(B-1)(d+1) v \frac{\partial}{\partial v}+(d+B) w \frac{\partial}{\partial w} . \\
X_{1}, Y_{1}, X_{2}, X_{6}(d=-1) . \\
X_{1}, Y_{1}, X_{3}, X_{6}(d=1) .\end{array}$ \\
\hline$\rho$ & Arbitrary & $X_{1}, Y_{1}, X_{7}=\rho \frac{\partial}{\partial \rho}+w \frac{\partial}{\partial w}$ \\
\hline $1 / \rho$ & $\begin{array}{l}\text { Arbitrary } \\
\mathrm{e}^{x}\end{array}$ & $\begin{array}{l}X_{1}, Y_{1}, Y_{8}=w \frac{\partial}{\partial t}+\rho^{2} v \frac{\partial}{\partial \rho}-\frac{1}{\rho} w \frac{\partial}{\partial v}+t \frac{\partial}{\partial w} . \\
X_{1}, Y_{1}, X_{5}(B=-1) \text {; infinite number of symmetries given by } \\
Y_{9}^{\infty}=\phi \frac{\partial}{\partial t}-2 \psi_{w} \frac{\partial}{\partial x}+\rho\left[\rho\left(v \phi_{w}+2 \psi_{w w}\right)+\psi_{w}\right] \frac{\partial}{\partial \rho}-\left[2 \phi_{w w}+v \psi_{w}+\phi_{w} / \rho\right] \frac{\partial}{\partial v}+\psi \frac{\partial}{\partial w}, \\
\text { where } \phi=\phi(t, w), \psi=\psi(t, w) \text { are arbitrary solutions of the linear system } \phi_{t}= \\
\psi_{w}, \phi_{w}=\psi_{t} ; \text { linearization by a point transformation. } \\
\text { Infinite number; linearization by a point transformation: } \\
x_{1}=t, x_{2}=w \text { (independent variables); } u_{1}=x, u_{2}=v, u_{3}=\rho \text { (dependent variables). } \\
\text { Infinite number; the system } \mathbf{L}(2.11) \text { is linear. }\end{array}$ \\
\hline$\rho^{3}$ & Arbitrary & Infinite number. \\
\hline
\end{tabular}

We look for point symmetries admitted by the system ER (3.13), which are nonlocal symmetries for both the Euler system E (2.10) and the Lagrange system EW (3.6).

Modulo the equivalence transformations (4.7), we find that such symmetries arise only in one case: $K(\rho)=\rho^{1 / 3}$, $f(x)=x$. In this case, the PDE system ER (3.13) admits an eight-dimensional symmetry algebra spanned by the symmetry generators

$$
\begin{aligned}
X_{1}= & \frac{\partial}{\partial t}-\frac{x^{2}}{2} \frac{\partial}{\partial r}, \quad X_{3}=\mathrm{e}^{t}\left(\frac{\partial}{\partial x}+\frac{\partial}{\partial v}+(1-t) x \frac{\partial}{\partial r}\right), \\
X_{4}= & \mathrm{e}^{-t}\left(\frac{\partial}{\partial x}-\frac{\partial}{\partial v}-(1+t) x \frac{\partial}{\partial r}\right), \quad X_{6}=-\frac{2}{3} x \frac{\partial}{\partial x}+2 \rho \frac{\partial}{\partial \rho}-\frac{2}{3} v \frac{\partial}{\partial v}, \\
Z_{1}=\frac{\partial}{\partial r}, \quad Z_{2}= & \mathrm{e}^{-t}\left[\left(x^{2}(2 t-1)+3\left(v^{2}-\rho^{-2 / 3}\right)+4 r\right) \frac{\partial}{\partial x}+6(x+v)\left(\frac{\partial}{\partial t}+\rho \frac{\partial}{\partial \rho}\right)\right. \\
& +\left(-x^{2}(2 t-3)+v(4 x-v)+3 \rho^{-2 / 3}-4 r\right) \frac{\partial}{\partial v} \\
& \left.+\left(x t\left(3\left(\rho^{-2 / 3}-v^{2}\right)-x^{2}(2 t+1)-4 r\right)+v\left(v^{2}-3 \rho^{-2 / 3}\right)-x\left(x^{2}+4 r\right)\right) \frac{\partial}{\partial r}\right], \\
Z_{3}= & \mathrm{e}^{t}\left[\left(x^{2}(2 t+1)-3\left(v^{2}-\rho^{-2 / 3}\right)+4 r\right) \frac{\partial}{\partial x}-6(v-x)\left(\frac{\partial}{\partial t}-\rho \frac{\partial}{\partial \rho}\right)\right.
\end{aligned}
$$




$$
\begin{aligned}
& \left.+\left(-x t\left(3\left(\rho^{-2 / 3}-v^{2}\right)+x^{2}(2 t-1)+4 r\right)-v\left(v^{2}-3 \rho^{-2 / 3}\right)-x\left(x^{2}-4 r\right)\right) \frac{\partial}{\partial r}\right] \\
Z_{4}= & \left(x\left(2 x^{2} t+4 r\right)-2 v\left(v^{2}+3 \rho^{-2 / 3}\right)\right) \frac{\partial}{\partial x}-3\left(v^{2}-x^{2}+9 \rho^{-2 / 3}\right) \frac{\partial}{\partial t}+6 \rho(x(v-x t)-2 r) \frac{\partial}{\partial \rho} \\
& +\left(2 x^{2}(x+v t)+4 v(r-x v)-6 x \rho^{-2 / 3}\right) \frac{\partial}{\partial v} \\
& +\left(-x^{4}\left(t^{2}+\frac{1}{4}\right)-\frac{3}{4} v^{2}\left(v^{2}+r \rho^{-2 / 3}\right)+2 x t v\left(v^{2}+3 \rho^{-2 / 3}\right)+\frac{9}{4} \rho^{-4 / 3}+4 r^{2}\right) \frac{\partial}{\partial r} .
\end{aligned}
$$

The symmetries $X_{1}, X_{3}, X_{4}, X_{6}, Z_{1}$ project onto point symmetries of the Euler system $\mathbf{E}$ (2.10) and the Lagrange system $\mathbf{E W}$ (3.6). The symmetries $Z_{2}, Z_{3}, Z_{4}$ are three new nonlocal symmetries for both the Euler system $\mathbf{E}$ (2.10) and the Lagrange system EW (3.6).

\section{Calculation of group invariant solutions arising from the Lagrange system EW}

In this section we present examples of exact solutions of the Euler system $\mathbf{E}$ (2.10) which arise as invariant solutions of the potential system $\mathbf{E W}$ (3.6) with respect to a potential symmetry of $\mathbf{E}$.

Since the stress $\sigma=K(\rho)$ is a function of density, we may equivalently rewrite the system $\mathbf{E W}$ (3.6) as

$w_{x}=\rho, \quad w_{t}=-\rho v, \quad K^{\prime}(\rho) \rho_{x}+\rho f(x)=\rho\left(v_{t}+v v_{x}\right)$.

We consider boundary conditions of the form

$v\left(x_{0}, t\right)=V(t), \quad \rho\left(x_{0}, t\right)=R(t), \quad w\left(x_{0}, t\right)=W(t)$.

In particular, for the problem of deformation of an elastic slab $x_{0}<x<L(t)$, attached at $x=x_{0}$, we use the boundary conditions

$v\left(x_{0}, t\right)=0, \quad \rho\left(x_{0}, t\right)=R(t), \quad w\left(x_{0}, t\right)=0$.

The latter boundary condition comes from the definition of the mass coordinate (potential variable): $w(x, t)=$ $\int_{x_{0}}^{x} \rho(s, t) \mathrm{d} s$.

\subsection{General strategy for finding invariant solutions}

The general method for finding invariant solutions is presented in detail in $[1,4]$, and is now summarized for the example of the potential system $\mathbf{E W}$ (5.1).

Let $G$ be a one-parameter Lie group of point transformations admitted by the potential system $\mathbf{E W}$. Let

$X=\xi \frac{\partial}{\partial x}+\tau \frac{\partial}{\partial t}+\eta^{v} \frac{\partial}{\partial v}+\eta^{\rho} \frac{\partial}{\partial \rho}+\eta^{w} \frac{\partial}{\partial w}$

be the infinitesimal generator of $G ; \xi, \tau, \eta^{v}, \eta^{\rho}$ and $\eta^{w}$ are functions of $x, t, v, \sigma, \rho$ and $w$.

The corresponding invariant solutions

$(v, \rho, w)=(V(x, t), R(x, t), W(x, t))$

of the potential system $\mathbf{E W}$ satisfy

$$
\left.X \cdot\left[\begin{array}{l}
v-V(x, t) \\
\rho-R(x, t) \\
w-W(x, t)
\end{array}\right]\right|_{(v, \rho, w)=(V(x, t), R(x, t), W(x, t))}=0
$$

as well as system $\mathbf{E W}$ (3.6). 
The system of first-order PDEs (5.6) yields characteristic ODEs

$$
\frac{\mathrm{d} x}{\xi}=\frac{\mathrm{d} t}{\tau}=\frac{\mathrm{d} v}{\eta^{v}}=\frac{\mathrm{d} \rho}{\eta^{\rho}}=\frac{\mathrm{d} w}{\eta^{w}} .
$$

The solution of (5.7) yields four arbitrary constants $A, B, C$ and $U$. These constants, expressed in terms of dependent and independent variables $x, t, v, \rho, w$, are invariants of the point symmetry defined by the generator (5.4):

$X A=X B=X C=X U=0$

The four invariants $A, B, C$ and $U$, together with a quantity $N(x, t, v, \rho, w)$ having the property

$X N=1$,

yield a set of canonical coordinates $(A, B, C, U, N)$ for the point symmetry (5.4). In these coordinates, the symmetry generator $X$ (5.4) corresponds to a translation symmetry

$X=\frac{\partial}{\partial N}$.

To find solutions of the PDE system EW (5.1) invariant with respect to the symmetry $X$ (5.4), one can proceed as follows.

- $\quad$ Express $x, t ; v(x, t), \rho(x, t), w(x, t)$ in terms of $U, N, A, B$, and $C$.

- In the PDE system EW (5.1), make a point coordinate transformation

$$
(x, t ; v(x, t), \rho(x, t), w(x, t)) \rightarrow(U, N ; A(U, N), B(U, N), C(U, N)) .
$$

(This transformation is non-singular, provided that invariants $A, B, C$ and $U$ are functionally independent.) As a result, one obtains an equivalent PDE system $\widetilde{\mathbf{E W}}$.

- Assume the invariance of the solution under the action of symmetry $X(5.8)$. Then $A(U, N)=A(U), B(U, N)=$ $B(U), C(U, N)=C(U)$. The PDE system $\widetilde{\mathbf{E W}}$ becomes a system of ODEs for corresponding invariant solutions.

- Solve the ODEs to find $A(U), B(U), C(U)$. The solution will involve several constants of integration.

- Using the solution $A(U), B(U), C(U)$, find expressions for the physical variables $v(x, t), \rho(x, t), w(x, t)$.

- Choose values of constants of integration to select a unique solution satisfying suitable boundary conditions (5.2).

- Compute the stress $\sigma(x, t)=K(\rho(x, t))$.

The resulting solution $(v(x, t), \rho(x, t), w(x, t), \sigma(x, t))$ of the potential system $\mathbf{E W}$ (3.6) directly yields a solution $(v(x, t), \rho(x, t), \sigma(x, t))$ of the Euler system $\mathbf{E}(2.10)$.

\subsection{Calculation of specific invariant solutions of the potential system $\mathbf{E W}$}

Finding closed-form solutions or useful reductions for compressible inhomogeneous isotropic materials is generally possible only for very specific classes of the strain energy function $W$ (see Sect. 2), as pointed out in [30]. For example, the case of axisymmetric deformations of solids in the absence of body forces gives rise to an equilibrium equation expressed in terms of a second order nonlinear ODE. It can further be reduced to a first order ODE for special forms of $W$.

Most closed-form exact solutions for compressible nonlinear elastic materials available in the literature are equilibrium solutions (restricted to a static situation), usually with the absence of body forces. In [31], a class of nontrivial "simple wave" solutions of the nonlinear 3D Lagrange system (2.5) was obtained for zero body forces and $W=W(\mathbf{F})$.

In the current paper, we consider BVPs for dynamical compressible nonlinear elasticity, and provide reductions of governing equations to a system of algebraic and ordinary differential equations that is solved exactly. The 
presented approach can be applied to a wide range of problems that a priori include different types of body forces $f=f(x)$, and include solutions corresponding to wave propagation problems.

We now find a family of exact dynamical solutions of the Euler system $\mathbf{E}$ (2.10) arising as group-invariant solutions of the Lagrange (potential) system EW (3.6).

From Table 2, we select a constitutive relation $\sigma=K(\rho)$ given by

$\sigma=K(\rho)=\frac{1}{2} \arctan \frac{1}{\rho}+\frac{1}{2} \frac{\rho}{\rho^{2}+1}$,

for the corresponding linear body force $f(x)=x$. The plot for the corresponding stress-strain relation $\sigma=K(1 / q)$ is shown in Fig. 2.

Corresponding to the above constitutive relation (5.9), we only consider the admitted potential symmetry

$Y_{4}=\frac{\mathrm{e}^{t}}{\rho}\left[\frac{\partial}{\partial t}+(v+\rho w) \frac{\partial}{\partial x}+(x+\rho w) \frac{\partial}{\partial v}-\rho\left(\rho^{2}+1\right) \frac{\partial}{\partial \rho}-\rho(x-v) \frac{\partial}{\partial w}\right]$.

We seek invariant solutions of the Lagrange (potential) system EW (3.6) with respect to symmetry $Y_{4}$, using the algorithm described in Sect. 5.1.

The characteristic equations (5.7) yield the following invariants:

$A=\frac{\rho \mathrm{e}^{t}}{\sqrt{1+\rho^{2}}}, \quad B=x^{2}-v^{2}+w^{2}, \quad C=\frac{x \rho-w}{\sqrt{1+\rho^{2}}}, \quad$ and $\quad U=\mathrm{e}^{t}(x-v)$.

The corresponding invariant solution has the form $A=A(U), B=B(U), C=C(U)$. The similarity variable $U=\mathrm{e}^{t}(x-v)$ effectively measures the deviation of velocity of a medium from a state $v(x, t)=x$. Thus the invariant solution describes a nonlinear deviation of a trivial "homogeneous stretching" solution $v(x, t)=x, \rho(x, t)=\mathrm{e}^{-t}$ of the Euler PDE system (2.10).

For convenience of notation, we choose as the translated canonical coordinate (see Sect. 5.1)

$M=-N=\frac{\mathrm{e}^{-t}}{\rho}$.

Expressing all dependent and independent variables in terms of canonical coordinates, we get

$x=\frac{C-U M^{2} A \pm \gamma M}{\sqrt{1-M^{2} A^{2}}}, \quad \mathrm{e}^{t}=\frac{A}{\sqrt{1-M^{2} A^{2}}}$,

$\rho=\frac{\sqrt{1-M^{2} A^{2}}}{M A}, \quad v=\frac{C-U / A \pm \gamma U}{\sqrt{1-M^{2} A^{2}}}, \quad w=-M U \pm \frac{\gamma}{A}$.

In (5.11), $\gamma=U^{2}+A^{2} B-2 U A C$. One may check that by definition, $A>0$ and $0<M^{2} A^{2}<1$, so the transformation (5.11) is always well-defined.

One can show that after the coordinate transformation (5.11) and the symmetry reduction $A(U, M)=A(U)$, $B(U, M)=B(U), C(U, M)=C(U)$, the system $\mathbf{E W}$ of three PDEs (3.6) reduces to a system of one algebraic and two ordinary differential equations given by

$\gamma=U^{2}+A^{2} B-2 U A C=0$,

$A^{2}(U) C^{\prime}(U)+A^{\prime}(U)(U-A(U) C(U))=0, \quad A(U) A^{\prime}(U)=U$.

The explicit solution of (5.12) is given by

$A(U)=\sqrt{U^{2}+\alpha^{2}}, \quad C(U)=\frac{1}{2} \frac{\alpha U+\left(U^{2}+\alpha^{2}\right)\left(\beta-\arctan \frac{U}{\alpha}\right)}{\alpha\left(U^{2}+\alpha^{2}\right)}$,

where $\alpha, \beta$ are constants of integration.

We now express the physical dependent variables $\rho, v, w, \sigma$ as functions of $x$ and $t$. From $(5.11)(\gamma=0)$, one obtains an equation that defines $v(x, t)$ implicitly:

$v(x, t)=\mathrm{e}^{t}\left(\frac{C(U)}{A(U)}-\frac{U}{A^{2}(U)}\right)$

where $A(U)$ and $C(U)$ are given by (5.13), and $U=\mathrm{e}^{t}(x-v(x, t))$. 
For every $x$ and $t$, the solution $v(x, t)$ of (5.14) can be found numerically. After $v(x, t)$ (and thus the similarity variable $U$ ) is determined, one readily finds

$\rho(x, t)=\frac{\sqrt{U^{2}+\alpha^{2}}}{\sqrt{\mathrm{e}^{2 t}-U^{2}-\alpha^{2}}}, \quad w(x, t)=\frac{v(x, t)-x}{\rho(x, t)}$.

\subsubsection{Properties of the invariant solution}

Before generating solution curves for the invariant solution given by (5.14)-(5.16), we outline several important features of this solution. First we recollect that we seek a solution describing a nonlinear deformation of an elastic slab $x_{0}<x<L(t)$, attached at $x=x_{0}$ (i.e., subject to a boundary condition $v\left(x_{0}, t\right)=0$.) When substituted in (5.14), this boundary condition yields

$\beta=0, \quad x_{0}=0$.

Hence $\beta=0$ in the following computations.

Since $w(x, t)=\int_{0}^{x} \rho(s, t) \mathrm{d} s \geq 0$, from (5.16), it follows that $v(x, t) \geq x$ for all $x, t$. Moreover, the density $\rho(x, t)$ given by (5.15) is defined if and only if $\mathrm{e}^{2 t}-U^{2}-\alpha^{2} \geq 0$. Hence

$|x-v(x, t)|=\left|U \mathrm{e}^{-t}\right| \leq 1-\alpha^{2} \mathrm{e}^{-2 t} \leq 1$,

i.e., the deviation of the material velocity from the "homogeneous" case $v(x, t)=x$ is bounded. Note that $0 \leq$ $\alpha \mathrm{e}^{-t_{0}}<1, t \geq t_{0}$.

We now study the solution of the implicit equation (5.14) that defines the velocity $v(x, t)$. For $\beta=0$, for an arbitrary choice of $\alpha$, solution curves have similar behaviour, and define a multi-valued function $v(x, t)$ ! Sample curves of $v(x, t)$ for $\alpha=2$ and times $t=1,1.2,1.4,1.6$ are shown in Fig. 3.

The velocity $v(x, t)$ is a three-valued function for $0 \leq x<x^{*}(t)$. The bifurcation point $x^{*}(t)$ can be characterized explicitly: setting $\mathrm{d} x / \mathrm{d} v=0$ in (5.14) and solving for $x$, one obtains

$v\left(x^{*}(t), t\right)-x^{*}(t)=\sqrt{\alpha \mathrm{e}^{-t}-\alpha^{2} \mathrm{e}^{-2 t}}$.

The system of two algebraic equations (5.14), (5.18) defines the position $x^{*}(t)$ of the bifurcation point and the physical velocity $v\left(x^{*}(t), t\right)$ there.

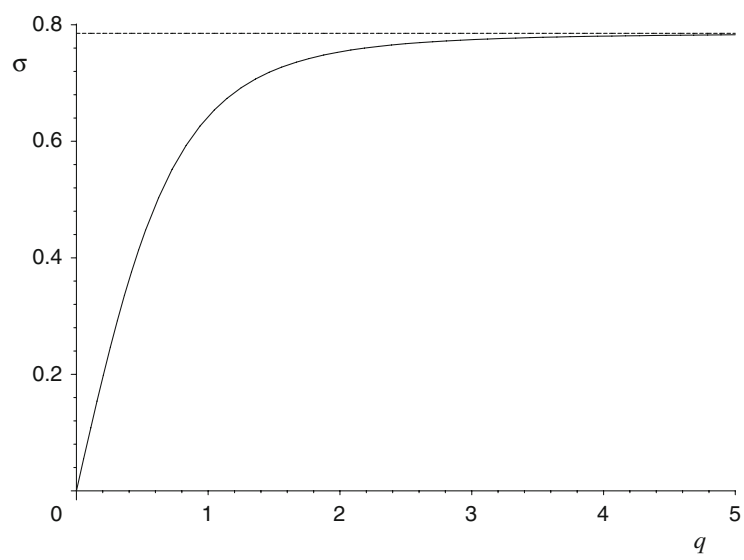

Fig. 2 Stress-strain curve for the constitutive relation $\sigma=K(\rho)$ given by (5.9)

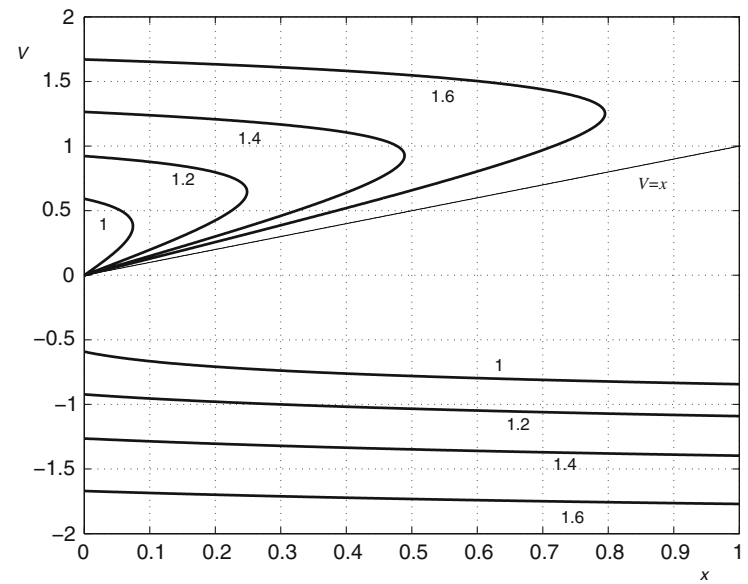

Fig. 3 Solution curves of the implicit equation (5.14) defining material velocity $v(x, t)(\alpha=2 ; t=1,1.2,1.4,1.6)$ 
Out of three possible values of $v(x, t)$ that arise from the implicit equation (5.14), only one branch is physical. Indeed, one may check that only the middle branch (closest to $v(x, t)=x$ ) satisfies the necessary condition (5.17) for the density to be a real-valued function.

Remark 5 It is important to note that the geometrical velocity of the bifurcation point $v^{*}(t)=\mathrm{d} x^{*}(t) / \mathrm{d} t$ is always greater than the physical velocity $v\left(x^{*}(t), t\right)$ at the bifurcation point.

In particular, the expression for the total mass between $x=0$ and the bifurcation point $x^{*}(t)$ (per unit area of the slab cross-section) is given by

$w\left(x^{*}(t), t\right)=1-\alpha \mathrm{e}^{-t}$,

which is an increasing function of time, in agreement with the previous remark.

The invariant solution we are interested in is defined for $0<x<x^{*}(t)$. If the initial length of the slab $L\left(t_{0}\right)$ is chosen $\left(0<L\left(t_{0}\right)<x^{*}\left(t_{0}\right)\right)$, then according to Remark 5 the solution is regular for all times.

\subsubsection{A particular example}

To produce specific solution curves, we choose $\alpha=2$ and the initial time $t_{0}=1$. We plot the graphs of field variables for $t=1,1.2,1.4$, and 1.6.

The positions of bifurcation points for these times are

$x^{*}(1) \approx 0.226, \quad x^{*}(1.2) \approx 0.483, \quad x^{*}(1.4) \approx 0.810, \quad x^{*}(1.6) \approx 1.209$.

We select the total mass of the slab (per unit cross-section) to be $M=0.25$; the length $L(t)$ is defined by the mass conservation condition $w(L(t), t)=M$. The total lengths of the slab and displacements of the material points corresponding to masses $0,0.2 M, 0.4 M, 0.6 M, 0.8 M, M$ are shown in Fig. 4.

Spatial distributions of the velocity, density and stress are shown in Figs. 5-7, respectively. In particular, the behaviour of spatial distributions of physical parameters, as well as the relation (5.18), suggest that the solution gets closer to the homogeneous solution $v(x, t)=x, \rho(x, t)=\exp (-t)$ as time increases.

\section{Concluding remarks}

In this paper we have considered the complete set of dynamic nonlinear elasticity equations in Lagrangian and Eulerian formulations. In the 1D case, we found a set of local conservation laws of the Euler system $\mathbf{E}(2.10)$

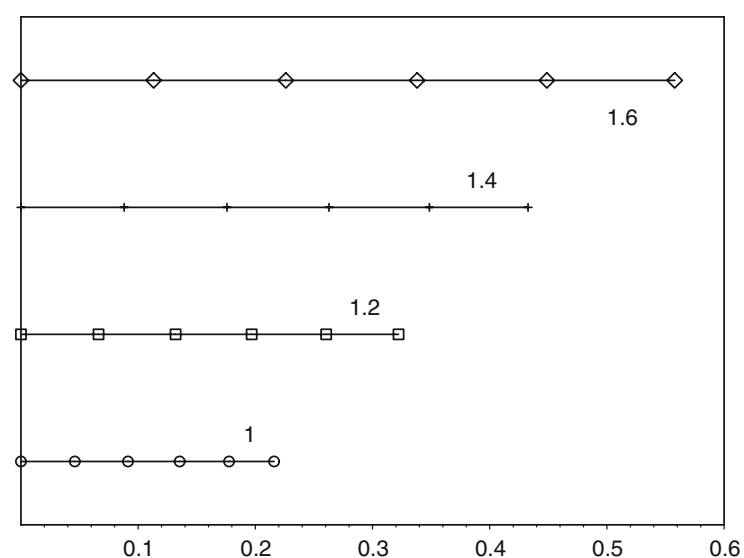

Fig. 4 Total lengths of the slab and displacements of the material points corresponding to masses $0,0.2 M, 0.4 M, 0.6 M, 0.8 M, M$, for times $t=1,1.2,1.4$, and 1.6. (Here $M=0.25$ )

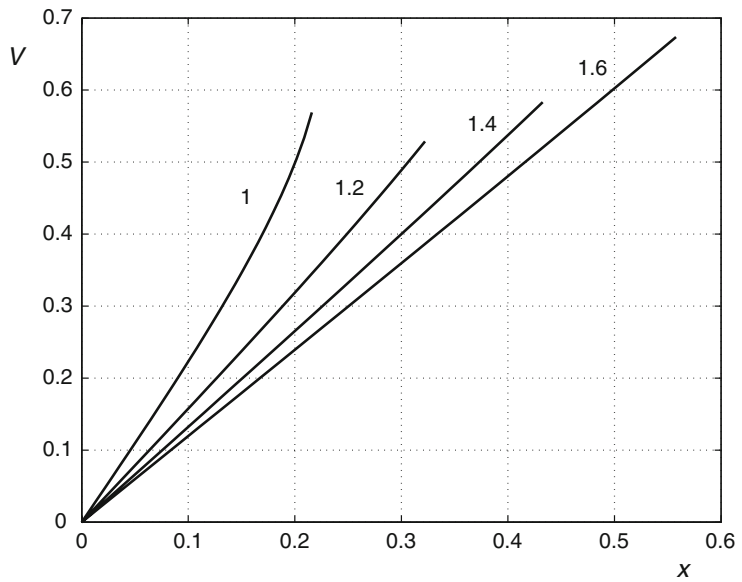

Fig. 5 Velocity distributions $v(x, t)$ for $t=1,1.2,1.4$, and 1.6 


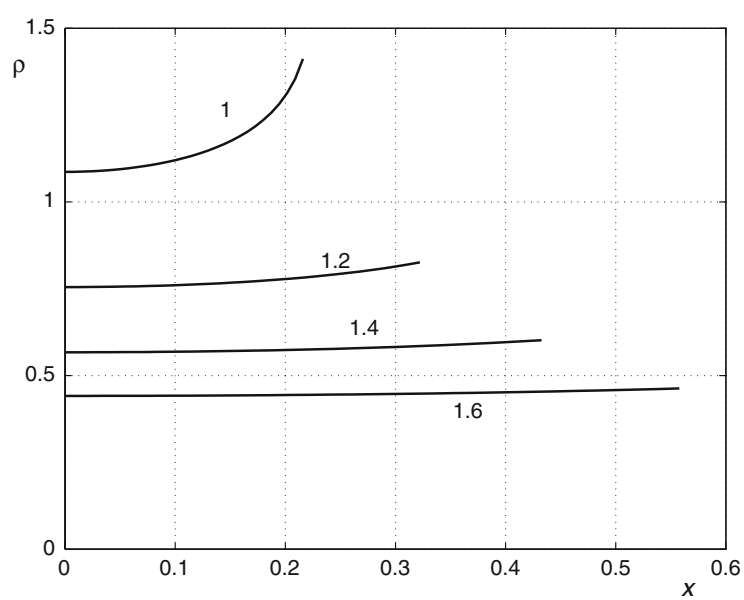

Fig. 6 Density distributions $\rho(x, t)$ for $t=1,1.2,1.4$, and 1.6

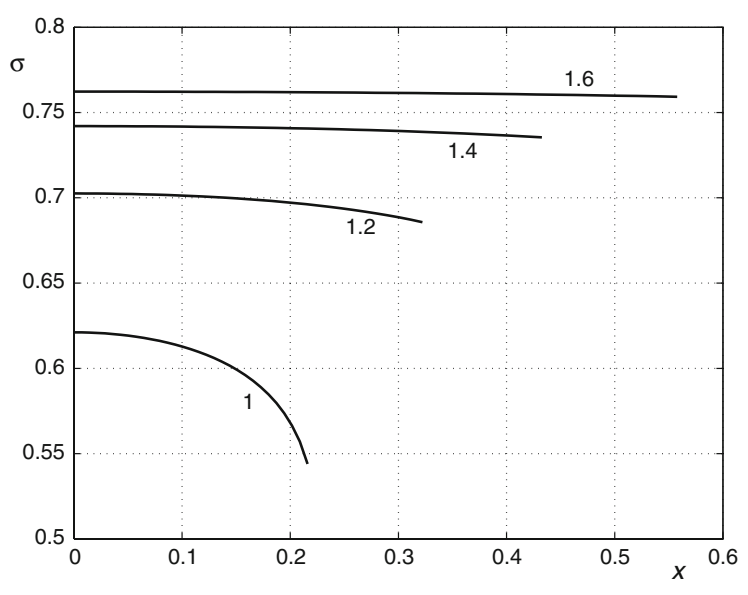

Fig. 7 Stress distributions $\sigma(x, t)$ for $t=1,1.2,1.4$, and 1.6

which hold for arbitrary constitutive functions $K(\rho)$ and $f(x)$. Through these conservation laws, we constructed a corresponding set (tree) of PDE systems nonlocally related to each other and equivalent to the Euler system.

Within our constructed tree, we have established the direct connection between the Lagrange and Euler systems. In particular, within our tree, the Lagrange system $\mathbf{L}$ (2.11) was shown to be invertibly equivalent to a potential system of the Euler system $\mathbf{E}$ (2.10). The Lagrange system arose from conservation of mass followed by a point transformation involving an interchange of a dependent variable (the potential variable associated with the conservation of mass) and an independent variable (spatial variable).

A complete point-symmetry classification was given for the Euler and Lagrange PDE systems. This yielded nonlocal symmetries for the Euler and Lagrange systems for several classes of constitutive functions. We also classified point symmetries of the potential system $\mathbf{E R}$ (3.13) of $\mathbf{E}$, which yielded nonlocal symmetries of $\mathbf{E}$ and $\mathbf{L}$.

Solutions of the Euler system of nonlinear elasticity that arise as invariant solutions of the Lagrange system but do not arise directly as invariant solutions of admitted point symmetries of the Euler system, were constructed for linear loads $f(x)=x$ and the stress-strain relation (5.9) for specific boundary conditions.

It would be worthwhile to complete the point-symmetry classification analysis for each of the nonlocally related systems ES, ERS, ERW and ERSW presented in this paper. Such an analysis may yield new nonlocal symmetries for the Euler and/or Lagrange systems for specific constitutive functions of interest. In turn, resulting invariant solutions may also yield new exact solutions for realistic BVPs.

Acknowledgements The authors thank the referees for very valuable suggestions that led to significant improvements in this paper. G.B. acknowledges support from NSERC (National Sciences and Engineering Research Council of Canada); A.F.C. is grateful for postdoctoral support from PIMS (Pacific Institute of Mathematical Sciences).

\section{References}

1. Bluman GW, Anco SC (2002) Symmetry and integration methods for differential equations. Springer, New York

2. Olver PJ (1993) Application of lie groups to differential equations. Springer, New York

3. Ovsiannikov LV (1982) Group analysis of differential equations. Academic Press, New York

4. Bluman GW, Kumei S (1989) Symmetries and differential equations. Springer, New York

5. Dorodnitsyn V, Winternitz P (2000) Lie point symmetry preserving discretizations for variable coefficient Korteweg-de Vries equations. Modern group analysis. Nonlin Dynam 22:49-59

6. Vassilev VM, Djondjorov PA (2003) Application of Lie transformation group methods to classical linear theories of rods and plates. Int J Sol Struct 40:1585-1614 
7. Ozer T (2003) Symmetry group classification of one-dimensional elastodynamics problems in nonlocal elasticity. Mech Res Comm 30:539-546

8. Ozer T (2003) Symmetry group classification for two-dimensional elastodynamics problems in nonlocal elasticity. Int J Eng Sci 41:2193-2211

9. Suhubi ES (2000) Explicit determination of isovector fields of equivalence groups for second order balance equations. Int J Eng Sci 38:715-736

10. Ozer T (2003) The solution of Navier equations of classical elasticity using Lie symmetry groups. Mech Res Comm 30:193-201

11. Suhubi ES, Bakkaloglu A (1997) Symmetry groups for arbitrary motions of hyperelastic solids. Int J Eng Sci 35:637-657

12. Bland DR (1969) Nonlinear dynamic elasticity. Ginn, Boston

13. Horgan CO, Murphy JH (2005) Lie group analysis and plane strain bending of cylindrical sectors for compressible nonlinearly elastic materials. IMA J Appl Math 70:80-91

14. Horgan CO, Murphy JH (2005) A Lie group analysis of the axisymmetric equations of finite elasttostatics for compressible materials. Math Mech Sol 10:311-333

15. Budiansky B, Rice JR (1968) Conservation laws and energy release rates. J Appl Mech 40:201-203

16. Hatfield GA, Olver PJ (1998) Canonical forms and conservation laws in linear elastostatics. Arch Mech 50:389-404

17. Yavari A, Marsden JE, Ortiz M (2006) On spatial and material covariant balance laws in elasticity. J Math Phys 47:042903

18. Anco S, Bluman G (2002) Direct construction method for conservation laws of partial differential equations. Part I: examples of conservation law classfications. Eur J Appl Math 13:545-566

19. Anco S, Bluman G (2002) Direct construction method for conservation laws of partial differential equations. Part II: general treatment. Eur J Appl Math 13:567-585

20. Bluman G, Cheviakov AF (2005) Framework for potential systems and nonlocal symmetries: algorithmic approach. J Math Phys 46:123506

21. Bluman G, Cheviakov AF, Ivanova NM (2006) Framework for nonlocally related PDE systems and nonlocal symmetries: extension, simplification, and examples. J Math Phys 47:113505

22. Ciarlet PG (1988) Mathematical elasticity. Volume I: three-dimensional Elasticity. Collection studies in mathematics and applications, vol 20. North-Holland

23. Ogden R (1997) Nonlinear elastic deformations. Dover

24. Truesdell C, Noll W (1965) The non-linear field theories of mechanics. In Handbuch der Physik, vol III/3. Springer, Berlin

25. Marsden JE, Hughes TJR (1983) Mathematical foundations of elasticity. Dover

26. Bluman G, Cheviakov AF (2007) Nonlocally related systems, linearization and nonlocal symmetries for the nonlinear wave equation. J Math An App 333:93-111

27. Bluman G, Cheviakov AF, Senthilvelan M (2008) Solution and asymptotic/blow-up behaviour of a class of nonlinear dissipative systems. J Math An App 339:1199-1209

28. Cheviakov AF (2007) Comp Phys Comm 176(1):48-61 (The GeM package and documentation is available at http://www.math. ubc.ca/ alexch/gem/.)

29. Wolf T (2002) Crack, LiePDE, ApplySym and ConLaw. In: Grabmeier J, Kaltofen E, Weispfenning V (eds) Computer algebra handbook. Springer, pp 465-468

30. Horgan CO (2001) Equilibrium solutions for compressible nonlinearly elastic materials. In: Fu YB, Ogden RW (eds) Nonlinear elasticity: theory and applications. Cambridge University Press, pp 135-159

31. Varley E (1965) Simple waves in general elastic materials. Arch Rat Mech Anal 20:309-328 